\title{
Reinterpreting Form Bionic Design and its Application in Design Innovation with Multiple Methods
}

\author{
Qiao Yang ${ }^{1, a}$ \\ ${ }^{1}$ Design Art and Media School, Nanjing University of Science and Technology, 21009, Nanjing, China
}

\begin{abstract}
This paper expounds the background and market demands of the application of Form Bionic Design (FBD) in design innovation. Then it analyses the advantages of FBD and existing misunderstandings on its definition and application, leading to the topic on how to correctly understand the classification of Bionic Design. Also it clarifies the significance of the integration of Bionic Design, proposing that FBD should follow the requirements of users and Design Hardpoints (DHPs). Then through a series of design cases it introduces the application of FBD compromised with several special methods, which includes Userexperiencing in simulation, Modular Design for system optimization and Keywords on style positioning. Meanwhile it respectively discusses the purpose, the function and particular application of each method, providing practical reference for its particular combination with FBD.
\end{abstract}

\section{Background}

The Internet has accelerated the modern economy, spawning an increasing number of new economic enterprises, such as Alibaba, Amazon, and eBay.

Driven by this trend, consumption desire has been greatly satisfied. Therefore the passion for novelty has extremely inflated, while the expectation for new design has risen dramatically. In order to meet booming market and changing consumption companies have constantly tried to apply extraordinary elements into design to ensure profits rising. Meanwhile, as the product cycle continues to shorten, designers have to create new design as soon as possible.

With the rapid development of science and technology, enterprises try to apply new technologies and new materials in innovation design. However, it normally takes longer than companies hope. Because, no matter how much value can be achieved from new application, it has to take a series of experimental tests and make certain improvements before it accesses to the market. Therefore, companies have to abandon the idea of applying new technologies or materials which cannot help cater the rapid growth of the market and the diversification of the consumption in such short term.

In order to address time-consuming problem of new applications, enterprises adopt many innovative design methods in the stage of product design and development, seeking to reduce costs and ensure effective profits while meeting the growing needs for innovation. NOKIA, SIEMENS and other major international companies have put forward the concept of "Design Library". Through this method they hope that enterprise designers and design teams can carry out forward-looking design exploration, and reserve concepts for future, so as to ensure them sufficient innovation to take the lead in the industry.

However, this method is extremely costly. It is unknown whether storage designs can really meet the future needs of enterprises and its practical effect cannot be seen until tomorrow. So for many small and mediumsized enterprises (SMEs) with limited cost, this method is not suitable. In contrast, Form Bionic Design (FBD) with natural advantages becomes one appropriate option, which can benefit the rapid and effective innovation with minimum expendure.

\section{Advantages of FBD}

The advantages of FBD are presented in the following aspects.

Firstly, the reference of FBD has a perfect natural model which organically integrates the structure and appearance to ensure good operation. Therefore it provides a quick-effective master for designers, who can quickly capture innovative ideas from natural ecosystems. Meanwhile it contributes to accurate positioning of appropriate referenced FBD model and highly reduces creative exploration.

Secondly, with numerous ecological prototypes in nature, FBD provides designers a rich bionic database with sufficient creative resources.

Thirdly, FBD reduces damages caused by less experimental time in quick application of new technologies and materials, which must be tested in a great number of regulation experiments before real application in products. So that is a long-term preparation which is also influenced by cost, production mode and many other factors. When these products enter into the market, it is more likely to see that new designs

*Corresponding author: ${ }^{a}$ Qiao Yang: mikejoe88@126.com 
may not satify customers nees which have already been changed. Fortunately, this problem can be solved by FBD, which can produce experimental prototypes extremely fast and provide sufficent resources and references for later design modification and development. In addtion, FBD can controls the cost effectively to enhance innovation under limitions of production and time.

\section{Reinterpret FBD and its application}

However, there are many misunderstandings on the definition and the application of FBD. Two of the most prominent ones are "separating form innovation from integrated design" and "lacking considerations for user needs and Design Hardpoints ".

\subsection{FBD and Integrated Design}

In some cases FBD has been completely seen as a pure form-simulation method, which not only separates exterior shape from functional requirements but also shields available inner structure in reference objects. In this way, new form will lack the support of the intrinsic functional-structure. Then it results in the loss of usability while designers must spend more time on building up matching functional structure according to the new appearance. Therefore, it not only loses initial time advantage of FBD, but also requires extra time to verify the rationality of new functional-structure influenced by the appearance change.

Thus, FBD is not just simple form mimicry. In FBD any form in nature can be used as if it can effectively unify form and functional structure. In fact, the reference prototype selected in FBD can provide rapid and intuitive application template, which is helpful for designers to quickly produce a suitable innovative model, effectively integrated with form, structure, and function.

Most misunderstandings are caused by a false understanding of bionic classification. It is well known that Bionic Design has many categories and the most commonly mentioned are functional bionics, structural bionics and form bionics.

However, since function, structure and form are unified in design and cannot be totally separated from each other, so why this classification has such independent name nominally? The truth is that one bionic concept is initially proposed from one angle belonging to one of these three aspects. For example, the Sea Harrier fighter typically belongs to functional bionics design. Its original intention is to realize the function of vertical take-off and landing, so that the carrier aircraft can take off and land quickly on a small aircraft carrier. Coincidentally, the function principle of sea piper flight just met this requirement. Another case is the gull-wing doors on sports car which is a typical example of form bionics. The original design purpose is to provide users' unusual visual experience. Through seagull-like doors, it satisfies the desire of hunting for novelty.
To sum up, function, structure and form in FBD have no strict boundaries in design. First of all, the function can be realized through the internal structure, while it builds up the carrier of appearance with external design to connect users. Secondly, the structure is not only the skeleton of the shape but also the benchmark for the smooth realization of target function. Finally, the form is the ultimate carrier of product design. Functionally, it is an external load module to protect internal structure and realize function. Visually, it is an external platform to guide usage and meet aesthetic needs.

\subsection{User requirements and Design Hardpoints}

In other cases user requirements and Design Hardpoints (DHPs) have been ignored in FBD.

Undoubtedly, the terminal object of design is to satisfy users. Therefore it is necessary to consider physical and psychological needs of users in FBD. Such user-centred considerations will help position appropriate reference of bionic form. Meanwhile, testing standards can be pre-set for later optimization and modification to promote design more reasonable.

DHPs in automotive design can be applied in other categories of product design. It is significant to define DHPs, including standards and requirements for function, size, material, human-factors, cost, process, installation and etc. In this way, the accuracy of design orientation can be guaranteed in the initial stage. Moreover, it can meet current production technical conditions and cost requirements [1]. In addition, it can set the applicable range and test standards for the later stage of FBD, reducing errors and saving costs.

Design Hardpoints is the general name of the control point (or coordinate), control line, control surface and control structure which are determined in the process of general layout design. It ensures the coordination and assembly of parts and meets styling requirements. This is the most important principle in design and selection of parts, accessories and body. It can make the components organized without mess. Also it is an important method of concurrent design, once determined then not easy to adjust. The DHPs is the soul of all design. [2]

According to the discussion above, it is obvious that FBD is not equal to pure form duplication. On the one hand, FBD can be applied effectively and reasonably as if the design satisfies DHPs. Otherwise its application will be totally failed. On the other hand, it is very crucial to develop adequate user-centred thinking and satisfy users' needs in FBD.

\section{FBD applied with multiple methods}

In order to meet the market demand for quick innovation and erase misunderstandings of FBD, it has integrated many methods with unique advantages to enhance the practical efficacy of FBD. Moreover this method helps increase the innovative value of design by meeting the changing needs of users timely.

The basic frame of FBD will still adopt traditional design process because it is beneficial for easy 
application of FBD in SMEs. In addition, the introduction of special methods in different stages of FBD can help update design and launch innovation step by step, according to actual conditions such as cost and producing level.

In the following part three different available methods applied in FBD are illustrated in details.

\subsection{User-experiencing in Simulation}

Simulation is the imitation of the operation of a realworld process or system. [3]

User-experiencing in simulation is used to discover useful and creative ideas by studying the adaptation and rationality of human-machine interaction in the simulation scenario. Meanwhile, by observing users' habits in usage and operating, designers can explore new functional models so as to provide reliable creative originals for FBD. Furthermore, it provides the testing criteria of user satisfaction for later optimization and final determination.

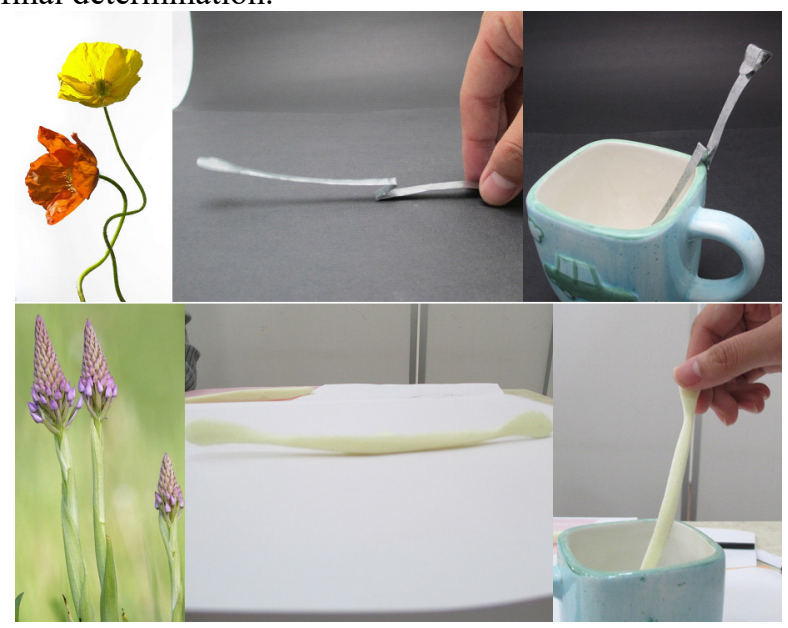

Figure 1. User-experiencing of coffee spoon

This method also helps to deduce reasonable and accurate aesthetic trends. According to the clear style positions, it can quickly promote a large number of styling designs, in which customers can really achieve enjoyment in vision. In Figure 1 the design of coffee spoon successfully combines User-experience in simulation with FBD to develop new concepts, which can meet demands of both usage and aesthetics.

\subsection{Modular Design}

Modular design, or "modularity in design", is a design approach that subdivides a system into smaller parts called modules or skids that can be independently created and then used in different systems. [4]

Modular design integrates the advantages of standardization and customization. This is usually due to the cost of putting up interfaces between modules. Under most circumstances a standard component is less expensive than a component designed and built for use in only one product. This lower cost is possible primarily because the standard component will be produced in higher volume, allowing greater economies of scale and more learning. [5]
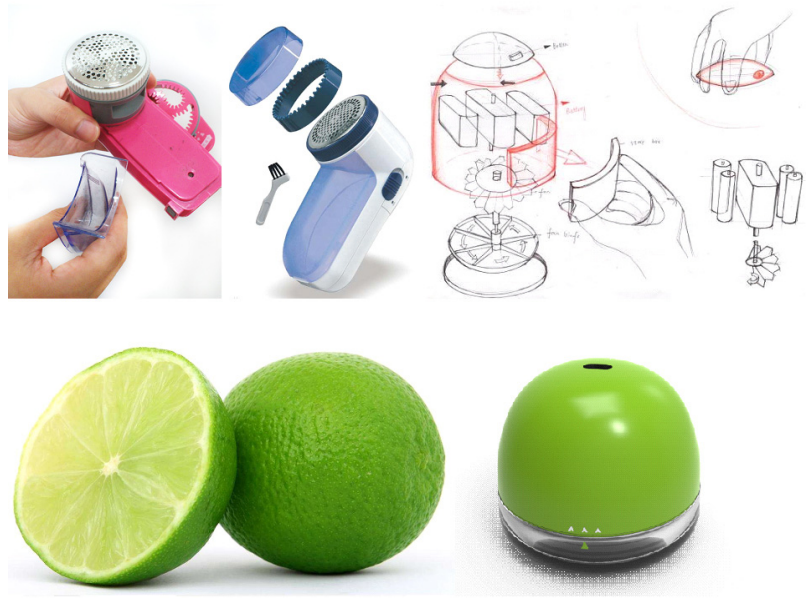

Figure 2. Modular design of Hair-Cleaner

First of all, the simplification of modular design helps quickly improve product innovation. By component optimization, novel appearance models can be obtained through minimal morphological changes. After that, suitable models can be found by comparing different new combinations. In this way, it is so quick to define DHPs accurately, which include motion space of functional units, mounting position of components, structure pattern and etc. All these DHPs provide a set of criterion for testing the reasonableness of subsequent designs.

Secondly, the serialization of modular design enables products to upgrade at different innovation levels through modular replacement. In order to accurately evaluating the rationality and feasibility of design, designers should pay more attention to technical parameters, such as original structure, material characteristics and parts' setting positions.

Thirdly, new technology implanted into a module can also stimulate new innovation opportunities.

In general, modular design is usually divided into three stages: functional module differentiation, module reorganization and model comparison. The details of each step are as follows:

Step 1. By analysing function and structure modules are differentiated functionally to establish the original model.

Step 2. Each functional group (or components groups which are needed to complete one function) is set up as a separate unit and then they are reorganized in different ways for creating new models.

Step 3. New models will be evaluated in a series of tests of the feasibility in many aspects, which includes engineering, man-machine, cost, material and processing difficulties. Then these models will be compared through functional experiments, man-machine analysis, cost analysis and etc. Finally, the most suitable module design can be found.

\subsection{Keywords on style position}

In FBD, how to ensure the selected reference to be applicable for design, especially for new form-styling? 
How to ensure the shape meets the aesthetic needs? This method, called as Keywords on style position, gives a valid answer which is presented in details as follows.

First of all, main function structure and HPs are determined according to the previous positioning. Next, a series of styling keywords that fit the user's preferences are listed one by one. Referring to the selected keywords, the related morphological reference objects are selected from the form-biomimetic point of view. Then, shapes of selected objects are refined and applied to FBD, which will continuously be optimized according to the predetermined standards of users' needs and HPs.

As figures shown in below, the motorboat design, a typical example of FBD, describes the specific application of Keywords on styling position.

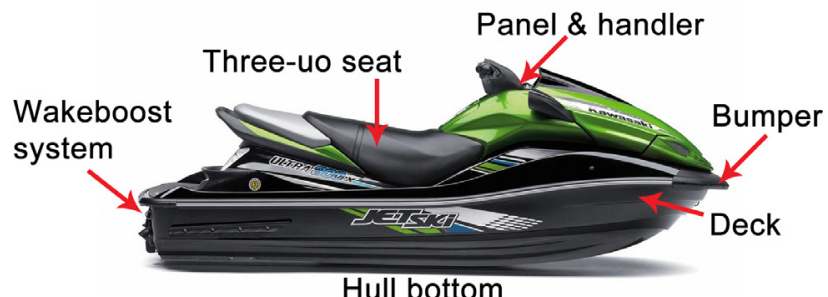

Figure 3. Modular of motorboat

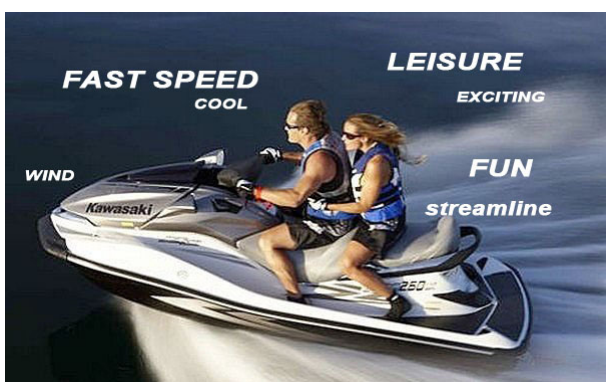

Figure 4. Keywords of users' favourite style

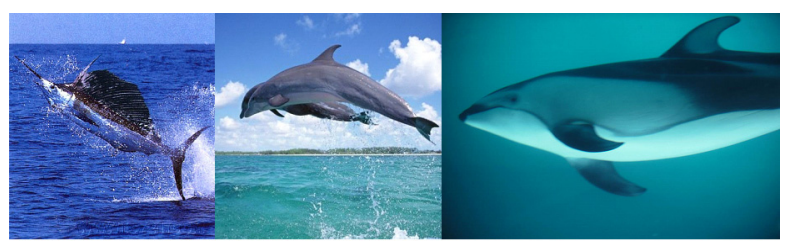

Figure 5. Relevant references
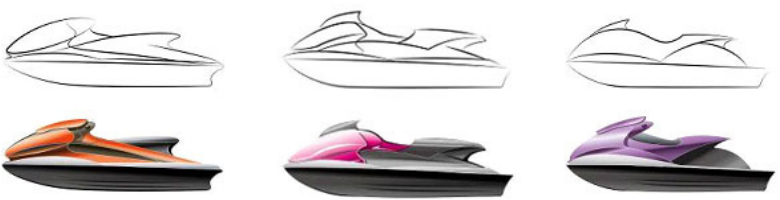

Figure 6. Shape refining and optimization

\section{Results}

FBD effectively promotes rapid design innovation and provides SMEs more opportunities to win the market while saving costs. FBD is not a simple form reproduction. In application it needs to consider more about users' requirements and HPs. Timely integration of other methods in different stages of FBD can effectively combine various advantages of multiple sides to improve the accuracy of innovation positioning and the practical value of new design.

\section{References}

1. X.Y. He, Y.F. Xie, Humanization Design (Jiangsu Fine Arts Publishing House, 2001)

2. C.Z. Sun, Y.C. Lei, Shanghai Auto 3, 33(2007)

3. J. Banks, J. Carson, B. Nelson, D. Nicol, DiscreteEvent System Simulation, (Prentice Hall, 2001)

4. F. Salimi, F. Salimi, A Systems Approach to Managing the Complexities of Process Industries, 234(Elsevier, 2017)

5. Ulrich.K, Res Policy 24(3), 431(1995) 